Relmecs, diciembre 2017, vol. 7, no. 2, e024, ISSN 1853-7863

Universidad Nacional de La Plata. Facultad de Humanidades y Ciencias de la Educación. Centro Interdisciplinario de Metodología de las Ciencias Sociales.

Red Latinoamericana de Metodología de las Ciencias Sociales

\title{
Complejidad y análisis sociológico. Un enfoque teórico y metodológico
}

\author{
Paolo Parra Saiani * \\ *Università degli Studi di Genova, Dipartimento di Scienze politiche, Italia | paolo.parra.saiani@unige.it
}

El hilo rojo que conecta los artículos de este número -variados y heterogéneos- es el tema de la complejidad, de manera manifiesta, como en los artículos de Rodríguez Zoya, Castro-Díaz, o de manera menos aparente, como en los de Pérez Rivas, de Moreno Moreno, de Hermida y de Lahire. Como afirma el mismo Lahire en su ensayo, lo que sorprende al observador de trabajos sociológicos es la extrema diversidad de tipos de objetos estudiados, diversidad presente también en este número de Relmecs, sea por el tipo de unidad de análisis, de escala temporal, o de importancia atribuida a dimensiones micro o macro.

Leonardo G. Rodríguez Zoya, en su "Problematización de la complejidad de los sistemas de pensamiento: un modelo epistemológico para la investigación empírica de los paradigmas”, recupera uno de los aspectos más relevantes de la obra de Edgar Morin: la problematización del paradigma y la organización de los sistemas de pensamiento. Asimismo, elabora una estrategia para superar una de las más fuertes limitaciones de su obra: la carencia de una metodología empírica para desarrollar el pensamiento complejo en el plano de la investigación científica. El autor problematiza la complejidad de los sistemas de pensamiento, elabora el concepto de problemas complejos, y luego propone un modelo epistemológico para la investigación empírica de los paradigmas. Un problema no es un dato de la realidad empírica, puesto que todo problema es el resultado de un proceso de problematización y, por lo tanto, una construcción emergente de una práctica social y cognitiva. Rodríguez Zoya empieza a partir la posición de Foucault, según la cual la problematización involucra un trabajo del pensamiento a través del cual éste elabora las condiciones acerca de "cómo y por qué ciertas cosas (una conducta, un fenómeno, un proceso) se convierten en un problema” (Foucault, 1988, p. 17). Si "la objetivación de una experiencia como problema implica juicios de valor”, un proceso de problematización es un proceso valorativo a través del cual un sistema observador juzga y evalúa una experiencia como problemática, y la "etiqueta 'problema complejo' asociada a un 'fenómeno empírico' alude a la construcción de un 'sistema observado' por parte de un 'sistema observador'”, en linea con el enfoque constructivo de Varsavsky $(1975,1982)$. Las preguntas cruciales -¿más deseable para quién?, ¿quién decide y define qué es lo deseable? - son preguntas a las cuales Rodríguez Zoya quiere responder resumiendo e ilustrando una investigación empírica para modelizar el sistema de creencias científicas de las ciencias de la complejidad y la simulación social. 
Ricardo Castro-Díaz, autor de "Epistemología y pragmatismo en el análisis de los sistemas complejos”, presenta una reflexión sobre los aportes y debate actual sobre la complejidad en la actuación investigativa de las ciencias sociales y humanas. El autor se focaliza especialmente en el análisis de sistemas complejos y su proceso de abstracción, construcción y estudio en el campo espacial y socioterritorial. El texto se plantea en dos partes principales contextualizando el pensamiento complejo como base fundamental para el análisis de sistemas, y luego se diserta sobre aspectos como la causalidad, estructura, evolución y dimensión. Finalmente, se constituye en un diálogo práctico para su ejecución en el abordaje del territorio. Los continuos adelantos tecnológicos para el análisis espacial determinan diversas formas de abordar el conocimiento sobre el territorio, tanto para el conteo de los elementos que lo componen como para sus cualidades relacionales. Esto se basa en la alta disponibilidad y uso de datos capturados usando múltiples herramientas (e.g. sensores remotos, bases de datos, software estadístico, etc.) que se circunscriben a los métodos usados por el investigador. Desde esta perspectiva, este artículo parte de un diálogo primario circunscrito a la reflexión crítica del hologramatismo propuesto por Morin ${ }^{1}$, que considera la problemática más allá de lo holístico, hacia una generalización representable, digerible y dinámica. Esto surge de una búsqueda -sin terminar- del pensamiento complejo y los aportes que pueden hacerse a la forma en que estudiamos, analizamos y “producimos” el espacio discutido en geografía, antropología o sociología. El objetivo sería borrar las fronteras que separan y dividen las ciencias en general (argumento que, como veremos, será planteado para Lahire también), para llegar a un análisis holístico que recupere la preinterpretación sobre la heterogeneidad de los elementos, la multiplicidad de sus conexiones y las características contextuales de la matriz territorial.

Diego Alfredo Pérez Rivas en su trabajo "La refutación del romanticismo y las condiciones epistémicas para la investigación sociohistórica en el neoilluminismo de Nicola Abbagnano” aborda el tema del estatus epistémico de la historia y la sociología, entre idealismo y positivismo. En la primera parte, el autor propone una breve reconstrucción de este debate; en la segunda, analiza la crítica de Abbagnano a ambos modelos, al ser considerados sistemas románticos. En la tercera y cuarta parte, analiza las condiciones epistémicas propuestas por el filósofo italiano para la historia y la sociología, resaltando la importancia de su refutación del romanticismo. Finalmente, evalúa si el esquema de Abbagnano es completo. Para Abbagnano, la principal condición epistémica de las ciencias sociales consistía en incluir el factor de la libertad humana (el riesgo, la problematicidad, la elección, etc.), renunciando a la pretensión de que los fenómenos sociales son procesos gobernados por leyes universales, necesarias y progresivas. La ciencia histórica tendría que recurrir a las fuentes primarias del conocimiento, a datos empíricos como objetos arqueológicos o inscripciones, sin especular sobre nociones románticas como el sentido de la historia. Lo mismo tendría que hacer la sociología, construyendo sus propias categorías de análisis y divorciándose de la filosofía y el derecho. La renuncia al romanticismo se podría interpretar como un primer paso hacia la rigurosidad epistémica tanto para la sociología como para la historia. La crítica de Abbagnano a los romanticismos fue vital en su concepción de las condiciones epistémicas para la sociología y la historia. Dicha crítica implicaba fundamentalmente la refutación del determinismo, pero es importante distinguirla de cualquier tipo de indeterminismo. Desde su punto de vista, el giro paradigmático a asumir era de carácter condicionalista-probabilista. Su propuesta incluía, por tanto, el uso de técnicas de observación, datos empíricos y herramientas matemáticas para describir la frecuencia de los fenómenos sociales, sin descartar el factor de la libertad humana. El esfuerzo sincrético de Abbagnano, que consistió en unir en una sola teoría sociológica los factores que componen a los fenómenos sociales, le parece bastante apropiado a Pérez Rivas para encarar el análisis de fenómenos complejos, puesto que evita una explicación unilateral de los fenómenos sociales, al elegir la descripción de probabilidades estadísticas, comportamientos y funciones recurrentes, pero considerando los factores de riesgo y la problematicidad de los propios fenómenos como factores condicionantes.

Mónica Cecilia Moreno Moreno, en su “Construyendo una noción de campesinos con un método”, quiere profundizar el trabajo de Orlando Fals Borda, quien subrayó la creatividad y apertura al cambio de los campesinos colombianos y modificó su imagen de pasividad y conservadurismo por naturaleza. El artículo se sostiene principalmente en la revisión de notas de campo y otro material del Archivo Satélite de Ciencias Humanas de la 
Universidad Nacional en Bogotá, y del Archivo Orlando Fals Borda del Archivo de Investigadores, ubicado en el Archivo Histórico de la Universidad Nacional de Colombia. Además, el capítulo se basa en los apartados sobre agricultura y nivel de vida de sus tesis de maestría y doctorado, artículos que escribió durante los años cincuenta e inicios de los años sesenta, y entrevistas hechas a campesinos y campesinas de la vereda Saucío. En la lectura de Fals no siempre los campesinos se negaban a implementar una práctica o herramienta moderna por su apego a la tradición: en sus notas de campo Fals da cuenta del rechazo a innovaciones sobre la base de motivaciones que ayudaban a comprender sus razonamientos y decisiones económicas. Tal forma de comprender las resistencias expone una noción de los campesinos como personas inteligentes, con capacidad analítica y abiertos al cambio, siempre y cuando existieran razones suficientemente justificadas para introducir prácticas y herramientas nuevas. Como escribiría Boudon varios años después, "un observador que emita el diagnóstico de la resistencia al cambio o la sumisión irracional del actor a las tradiciones, solo daría fe de su ignorancia" (1984, pp. 60-61).

Mariano Hermida, en "Los indicadores de la dimensión social del desarrollo sostenible, el caso de Tierra del Fuego”, aborda la dinámica social involucrada en el desarrollo sostenible, y profundiza sobre el recorrido teórico metodológico en relación con el concepto analizado. Como ejemplo analiza las dinámicas sociodemográficas y socioeconómicas de la provincia de Tierra del Fuego, y presenta los indicadores sociales propuestos con base en distintos criterios, principalmente los respectivos al abordaje teórico y a la calidad y disponibilidad de los datos. El desarrollo local sostenible es un concepto complejo, por lo que una mirada holística basada en un modelo sistémico como marco ordenador de los indicadores parece ser la opción más indicada. Para observar las transformaciones en la dimensión social y su interrelación con los restantes subsistemas, Hermida ha seleccionado como marco conceptual ordenador un modelo sistémico basado en la perspectiva socioecológica propuesta por la CEPAL para la Evaluación de la Sostenibilidad de América Latina y el Caribe (ESALC), ya utilizado por el Ministerio de Ambiente y Desarrollo Sustentable de la Nación para el Sistema de Información del Desarrollo Sostenible de Argentina (SIDSA). También señala que gran parte de los indicadores debieron ser calculados por el mismo autor: este mismo aspecto puede ser considerado como indicador de la baja de utilización de la información estadística que se hace en el ámbito de la provincia, y Hermida nos invita a pensar en la necesidad de generar una “cultura estadística” como base de las políticas públicas.

Bernard Lahire, en su ensayo “Monde pluriel: pourquoi les individus font-ils ce qu’ils font?”, sigue trabajando sobre el tema de la hiperespecialización del trabajo en las ciencias humanas y sociales $\stackrel{2}{ }$, que conduce a una fragmentación en disciplinas separadas y en sectores especializados dentro de cada disciplina, y a las consecuentes dificultades que encuentran los científicos en sus estudios sobre los efectos de la diferenciación social de las actividades. Aquí la complejidad se presenta cuasi como paradójica: de un lado, una diferenciación social que afecta todos los sectores de la sociedad y, por consecuencia, también el trabajo científico; del otro, la misma diferenciación social del trabajo científico que hace siempre más difícil conservar una concepción compleja de los individuos en la sociedad, evitando, al mismo tiempo, "las formas empíricamente perezosas y teóricamente pretenciosas de los pensamientos de la complejidad". Lahire, como ya había hecho en sus trabajos anteriores (1998, 2002 y 2012), defiende la validez general del modelo teórico disposicionalista y contextualista en el proceso de análisis social, y plantea que su fórmula "Pasado incorporado + Contexto de acción presente = Prácticas" supera - de alguna manera incorporándolas- categorías como la de habitus y de campo, presentes en la igualmente famosa ecuación de Bourdieu según la cual “Habitus (Capital) + Campo = Práctica”.

El volumen concluye con una reseña del libro de Ana Carolina Arias y Matías David López, Indisciplinas. Reflexiones sobre prácticas metodológicas en ciencias sociales, presentado por Ramiro Segura. Coherentemente con el hilo rojo de ese número, en Indisciplinas -un libro que reúne jóvenes investigadores/as en formación de la Universidad Nacional de La Plata- se presentan experiencias de investigación que han involucrado una pluralidad de métodos de trabajo. 


\section{Notas}

1 “En el holograma, cada parte contiene la información del todo. No la contiene, por lo demás, totalmente; pero la contiene en gran parte, lo que hace que efectivamente podamos romper la imagen del holograma, reconstituyéndose otros micro-todos fragmentarios y atenuados. [...] En el lenguaje, el discurso toma sentido en relación a la palabra, pero la palabra sólo fija su sentido en relación a los discursos en los que se encuentra encadenada. [...] Los problemas de organización social sólo pueden comprenderse a partir de este nivel complejo de la relación parte-todo” (Morin 2004, pp. 6-7).

$\underline{2}$ Véase, por ejemplo, Lahire (2005).

\section{Bibliografía}

Boudon, R. (1984). La place du desordre. Critique des théories du changement sociale. Paris: Presses Universitaires de France.

Bourdieu, P. (1979). La Distinction. Critique sociale du jugement. Paris: Minuit.

Foucault, M. (1988). On problematization. The History of the Present, 4, 16-17.

Lahire, B. (1998). L'Homme pluriel. Les ressorts de l'action. Paris: Nathan.

Lahire, B. (2002). Campo, fuera de campo y contracampo. Colección Pedagógica Universitaria. 37-38. (En línea) http://www.uv.mx/cpue/coleccion/N 3738/H\%20Lahire\%20campo\%20contracampo.pdf >

Lahire, B. (2005). Misère de la division du travail sociologique: le cas des pratiques culturelles adolescentes. Education et sociétés, 16(2), 129-136. doi:10.3917/es.016.0129.

Lahire, B. (2012). Monde pluriel. Penser l'unité des sciences sociales. Paris, Le Seuil.

Morin, E. (2004). La epistemología de la complejidad. Gazeta de antropología, 20, art. 2, 1-14.

Varsavsky, O. (1975). Marco Histórico Constructivo para estilos sociales, proyectos nacionales y sus estrategias. Buenos Aires: Centro Editor de América Latina.

Varsavsky, O. (1982). Ideas básicas para una filosofía constructiva. En O. Varsavsky (Ed.), Obras Escogidas (pp. 365-413). Buenos Aires: Centro Editor de América Latina. 